\title{
THE FORMATION MECHANISM, INFLUENCING FACTORS AND PROCESSING CONTROL OF STRAY GRAINS IN NICKEL-BASED SINGLE CRYSTAL SUPERALLOYS
}

\author{
Yafeng Li, Lin Liu, Taiwen Huang, Dejian Sun, Jun Zhang, Hengzhi Fu \\ State Key Laboratory of Solidification Processing, Northwestern Polytechnical University \\ Xi'an 710072, PR China
}

Keywords: Directional solidification, Single crystal, Stray grain, Mechanism, Influencing factors, Control methods

\begin{abstract}
The thermal profile of a directionally solidifying nickel-based single crystal (SX) turbine blade was simulated using a Procast finite element technique. The result showed that the local thermal profile in the platform is wave-shaped rather than a planar or consistent concave. Such a distributed thermal profile forms an isolated thermal undercooling zone in the platform end and affects the dendritic branching process into the platform region. Based on the thermal and grain growth analyses, the criterion of stray grain formation was proposed as a competition process on the time of the dendritic branching from the airfoil to the platform end and the cooling of the platform end to the heterogeneous nucleation temperature. The influencing factors, namely the withdrawal rate, the directional solidification method, the platform dimension, the alloy composition and the crystal orientation on stray grain formation were then systematically investigated according to the criterion. Several stray grain (SG) controlling techniques with different mechanisms were proposed and analyzed based on the thermal evolution process.
\end{abstract}

\section{Introduction}

Nickel-base superalloy turbine blades serving as the main power extraction components in combustor of the turbine engine are routinely fabricated in single crystal form for its excellent creep, fatigue and oxidation/corrosion resistance [1]. To further extract power from hot section for the higher maneuverability of the modern engines, the elevated inlet gas temperature requires higher temperature tolerated turbine blade to be utilized, which is achieved by optimizing the inner cooling passages and using the refractory elements revised alloys with higher melting point.

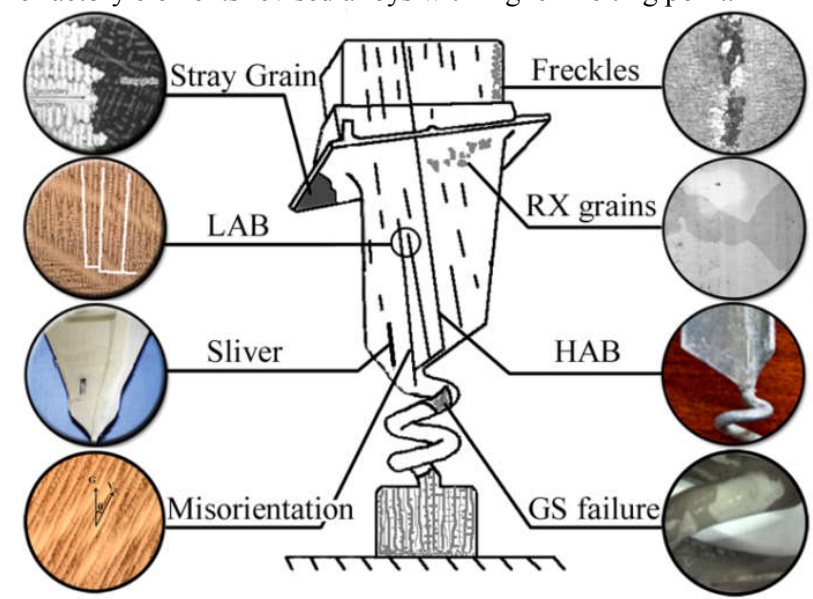

Figure 1. Typical anomalies in single crystal castings (modified from [2]). LAB: low angle boundary; RX: recrystal; HAB: high angle boundary; GS: grain selection.

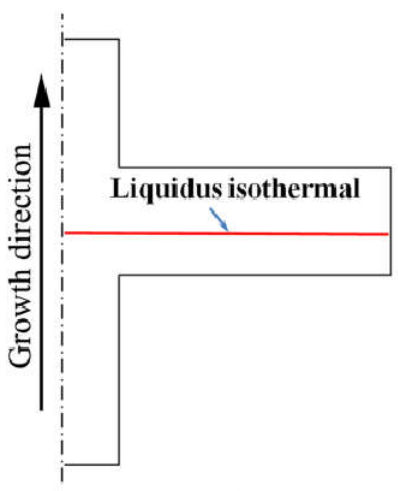

(a)

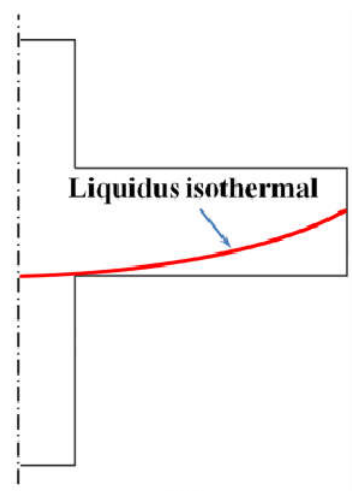

(b)
Figure 2. Schematic illustration of the liquidus isothermal shape in platform region of a turbine blade; (a) assumed planar in [3] and (b) assumed consistent concave in [4].

Accompanying these improvements, however, are the more stringent process requirements, more severe segregation and the increased susceptibilities of anomalies occurrence. Performancelimiting crystal anomalies, such as freckle chains, low angle boundaries (LABs), and stray grains are often observed in SX turbine blades (Figure1). Among all anomalies, SG is the most detrimental as this may introduce an unexpected high angle boundary (HAB) to the matrix grain resulting in crack initiation in service. The harm of this anomaly is enhanced particularly in the case where alloys with low levels of grain boundary strengtheners are used for casting SX components.

Previous research on the mechanism [3, 4], the influencing factors [3-9] and the control methods [4, 10, 11] of SG in the platform are all based on an assumed fact that the liquidus isothermal in the platform region is either planar or consistent concave (Figure2). Bussac [3] derived a theoretical model to predict the SG formation under the assumption that the thermal profile is planar. Meyer [4] depicted the SG formation as a nucleation process occurring in a thermally undercooled zone caused by the consistently concaved liquidus isotherm. Yang [5] applied a combined cellular automaton-finite difference (CA-FD) model to investigate the isothermal inclination angle on SG formation where the planar isothermal was set to converge or diverge to the platform base. Due to the abruptly changed configuration of the platform, the shape of the thermal profile is more complex than simply a planar or consistently concave, leading to a much more complicated dendritic branching process and a resulting different SG formation criterion.

In this study, the thermal profile was first simulated and characterized followed by an analysis of the dendritic branching process. Thereafter, a revised criterion of SG formation was 
proposed. Based on the criterion, the influencing factors on SG formation were systematically investigated and several SG controlling techniques were discussed.

\section{Procedure}

\section{Experimental Process}

Blade Model. A simplified model of a typical turbine blade consisted of a rectangle airfoil section with confirmed dimension and a variably sized platform (Figure 3 (a)) was used to fabricate SXs. Among four types of platform dimensions listed in Table I, type 1 was used as the base to investigate the SG mechanism, the influencing factors on SG formation and the SG controlling methods. The other three were used to investigate the platform dimension on SG formation. In order to investigate the crystal orientations on SG formation, a seeding-grain selection technique [12] was used to precisely control crystal orientation as illustrated in Figure 3 (b) and (c).

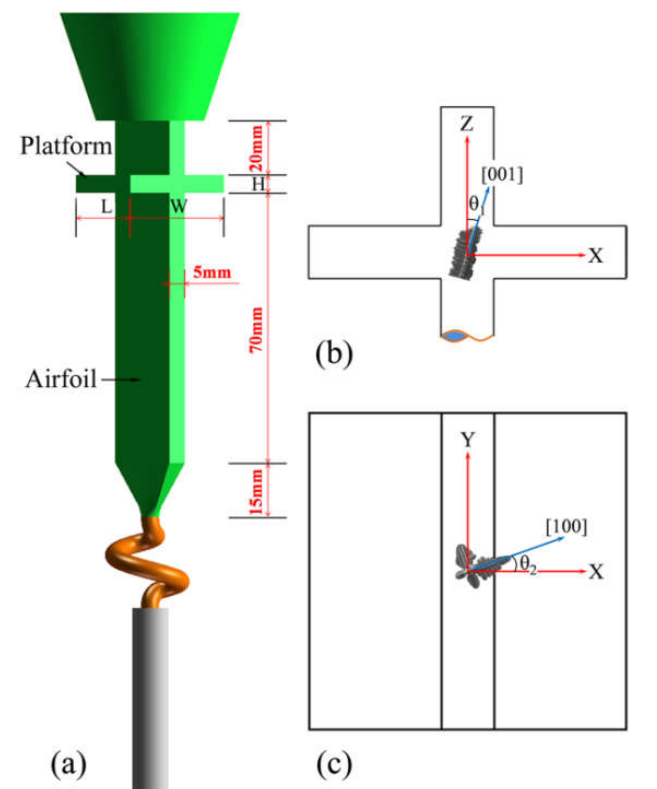

Figure 3. Schematic of the simplified turbine blade assembled with a spiral grain selector (a) and the default coordinate system and relative deviation angles $\theta_{1}$ and $\theta_{2}$ seen from front-view (b) and top-view (c). $\theta_{1}$ : the deviation angel of [001] to $\mathrm{Z}$ axis; $\theta_{2}$ : the torsion angle of [100] to $\mathrm{X}$ axis.

Table I Platform dimensions used in study

\begin{tabular}{cccc}
\hline \multirow{2}{*}{ Platform type } & \multicolumn{3}{c}{ Dimension } \\
\cline { 2 - 4 } & Length $(\mathrm{mm})$ & Width $(\mathrm{mm})$ & Height $(\mathrm{mm})$ \\
\hline Type 1 & 30 & 30 & 5 \\
Type 2 & 40 & 30 & 10 \\
Type 3 & 46 & 36 & 10 \\
Type 4 & 52 & 42 & 10 \\
\hline
\end{tabular}

Molds and Seeds Preparation. Each component of the casting was prepared by injecting molten wax into corresponding mold. These wax components were then assembled to the entire casting like the schematic drawing shown in Figure 3 (a). The assemblies were then coated with ceramic slurry consisting of a binder and filler material (80\% alumina and $20 \%$ silica) with additions of wetting and antifoaming agents to improve slurry rheology. Then the surface was stuccoed with coarse ceramic grit to adhere to the wet coating and harden it. To build up the shell thickness, six layers were successively built, including the primary coating, and 2-hour intervals were used to dry every successive layer. After the shell was constructed, the molds were then dewaxed in a steam autoclave, and subsequently fired at a temperature of $800{ }^{\circ} \mathrm{C}$ to partially sinter the ceramic shell.

SX rods were pre-fabricated to prepare seeds using spiral grain selection technique. The orientations of these SX rods were determined by measuring the sectioned samples using X-ray diffraction technique. According to the measured orientations, the rods were then cut into cylinders with desired dimensions and orientations. The information of the orientation was marked in the bottom cross section of the seeds. A subordinate coordinates in accordance with the default coordinates in platform region (Figure 3 (b), (c)) were set in the bottom plane of the mold. By adjusting seeds using the marks of the seeds and the subordinate coordinates of the mold, the relative orientation of seeds to platform was satisfied. The seeds were then fixed in mold.

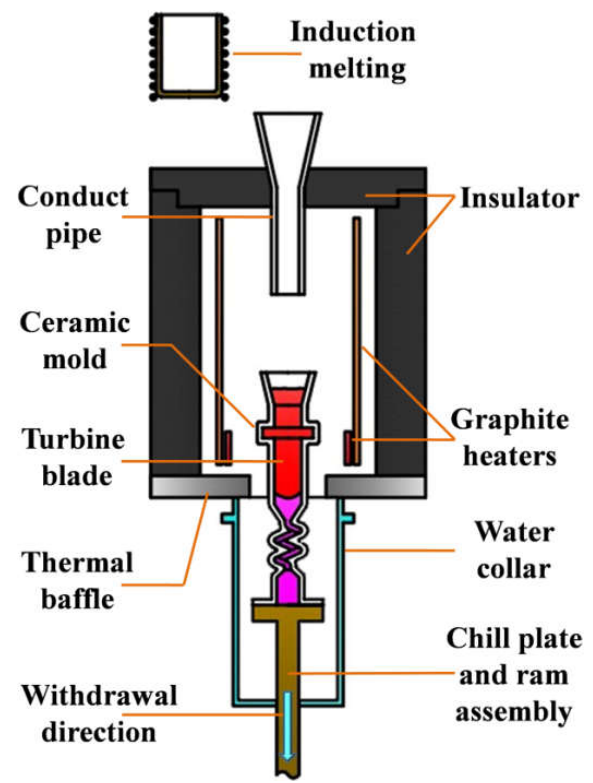

Figure 4. Schematic diagram of the furnace chamber.

Alloys Used In Study. The alloys used in study are listed in Table II. The first generation alloy DD403 was used as the base alloy to investigate the SG mechanism, the influencing factors on SG formation and the SG controlling methods. Alloy 2- Alloy 7 were aimed to investigate the alloy composition on SG formation. Among these alloys, Alloy 2 and Alloy 3 were designed for investigating the element Rhenium (Re) on SG formation. Alloy 4 - Alloy 7 were designed for investigating the trace elements Carbon (C) and Boron (B) on the SG formation.

Casting Process. The SX castings were prepared using a modified Bridgman directional casting furnace capable of operating in both radiation cooling and liquid metal cooling (LMC) modes, schematically shown in Figure 4. During directional solidification, 
the furnace chamber was first evacuated to a partial pressure of approximately $10^{-2} \mathrm{~Pa}$. The ceramic mold with seed prefixed mounted on a water-cooled copper chill plate was then preheated to $1550{ }^{\circ} \mathrm{C}$ by graphite heating elements. After the ingot was melted, the liquid melt of $1500{ }^{\circ} \mathrm{C}$ was poured into the preheated mold cavity and held for $10 \mathrm{~min}$ to stabilize. Finally, the ceramic mold was withdrawn out from the furnace at a predetermined withdrawal rate. To investigate the influences of different variables on SG formation, the values of different parameters were set in ranges around the baseline, as listed in Table III. The detailed settings of these parameters are discussed in the Results and Discussion.

Table II Compositions of the alloys used in study (wt.\%)

\begin{tabular}{cccccccccccc}
\hline Alloy & $\mathrm{Cr}$ & $\mathrm{Co}$ & $\mathrm{Mo}$ & $\mathrm{W}$ & $\mathrm{Re}$ & $\mathrm{Al}$ & $\mathrm{Ta}$ & $\mathrm{Hf}$ & $\mathrm{C}$ & $\mathrm{B}$ & $\mathrm{Ni}$ \\
\hline DD403 (Baseline) & 9.5 & 5.0 & 3.8 & 5.2 & & 5.9 & $2.1 \mathrm{Ti}$ & & & & $\mathrm{Bal}$ \\
Alloy 2 (3.16Re) & 3.01 & 11.6 & 1.02 & 5.90 & 3.16 & 6.11 & 7.73 & 0.04 & & & $\mathrm{Bal}$ \\
Alloy 3 (6.04Re) & 2.99 & 11.8 & 1.03 & 5.85 & 6.04 & 6.08 & 7.79 & 0.09 & & & $\mathrm{Bal}$ \\
Alloy 4 (0.085C) & 5.05 & 12.0 & 1.03 & 6.00 & 5.08 & 5.99 & 8.14 & 0.09 & 0.085 & & $\mathrm{Bal}$ \\
Alloy 5 (0.130C) & 5.08 & 11.9 & 1.01 & 5.83 & 4.95 & 5.96 & 7.86 & 0.08 & 0.13 & & $\mathrm{Bal}$ \\
Alloy 6 (0.005B) & 4.95 & 11.9 & 1.01 & 5.80 & 4.96 & 6.03 & 7.96 & 0.08 & 0.072 & 0.005 & $\mathrm{Bal}$ \\
Alloy 7 (0.014B) & 4.94 & 11.8 & 1.01 & 5.77 & 4.87 & 5.99 & 7.85 & 0.07 & 0.078 & 0.014 & $\mathrm{Bal}$ \\
\hline
\end{tabular}

Table III Baselines and the range of investigated values for studies on SG formation

\begin{tabular}{cccc}
\hline Parameter & Units & Baseline & Range \\
\hline Alloy & & DD3 & Alloy 2 -- Alloy 7 \\
Platform geometry & $\mathrm{mm}$ & Type 1 & Type 2, Type 3, Type 4 \\
Direction solidification method & & High rate solidification (HRS) & Liquid metal cooling (LMC) \\
SX orientation $\left(\theta_{1}, \theta_{2}\right)$ & $\circ$ & $(0,30)$ & $(15,0),(0,0),(0,15),(0,30),(0,45)$ \\
Withdrawal rate & $\mathrm{mmmin}^{-1}$ & 6,9 & $3,12,9-3$ \\
\hline
\end{tabular}

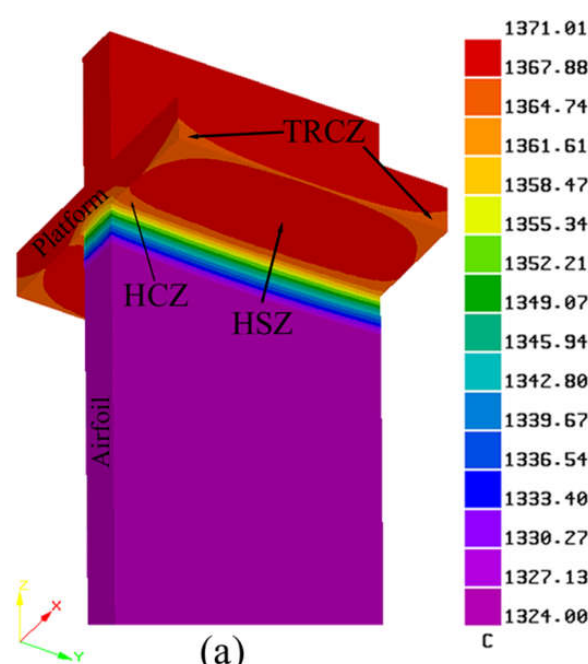

(a)

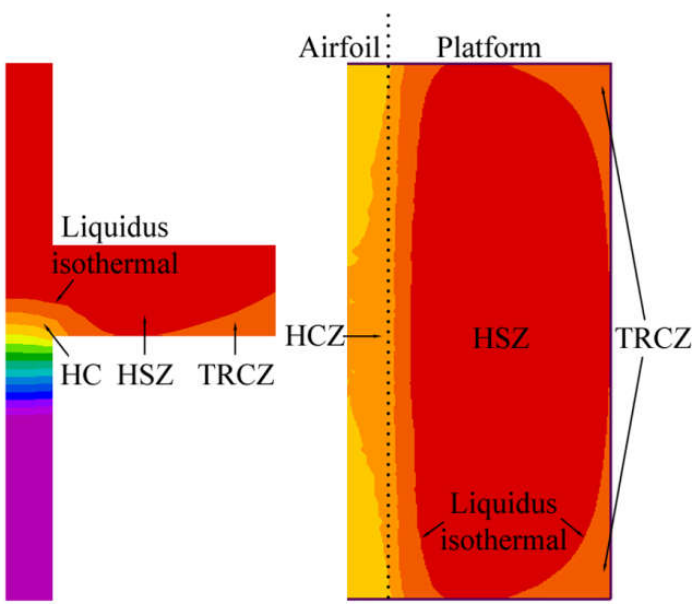

(c)

Figure 5. Simulated thermal profile in the platform region of a solidifying turbine blade (a) and the enlarged section seen from front-view (b) and top-view (c). $\left(V=6 \mathrm{~mm} \mathrm{~min}^{-1}, t=1658 \mathrm{~s}\right)$. HCZ: heat conduction zone; HSZ: hot spot zone; TRCZ: thermal radiation cooling zone

\section{$\underline{\text { Simulation Process }}$}

Solidification of the blades was simulated using finite element analysis with ProCAST in order to exhibit the thermal profile in directional solidification process. Such simulations are relatively routine. The salient points of the calculations are highlighted below:

(1) The simulation models of the furnace and the blade are constructed according to the actual dimensions. The thickness of the ceramic shell adhering to the finite element dispersed blade was assigned $7 \mathrm{~mm}$ from actual measurement.
(2) The heat transfer was assumed to be heat conduction and radiation with heat convection being ignored in HRS technique, while in LMC process, the heat convection has been considered. The coefficients of thermal emissivity, thermal conductivity and heat convection were gained partly from reference $[13,14]$ and partly from experiments.

(3) The thermophysical parameters of the used alloys were calculated by the commercial software JMatPro (Trade Mark of Sente Software Ltd. Surrey Technology Center, Surrey GU2 7YG, United Kingdom).

(4) The simulated thermal results were validated through thermocouple instrumented experiments. 


\section{Results and Discussion}

\section{Mechanism of Stray Grain Formation}

To investigate the mechanism of SG formation, the local thermal profile in platform region was simulated and compared with the planar or concave shaped thermal profile to investigate the dendritic branching process under these different thermal conditions. Based on these analyses, a revised criterion of SG formation was proposed.

Thermal and Dendritic Evolution Process. The local thermal profile in the platform region of a solidifying blade is shown in Figure 5. The main conclusions can be drawn as follows:

a. The liquidus isothermal profile is wave-shaped with a high temperature valley stationed in the middle section of the platform which constitutes a HSZ.

b. The HSZ separates the TRCZ in the platform end from the $\mathrm{HCZ}$ near the airfoil section by discontinued liquidus isothermal.

The shape of the actual thermal profile in platform region is rather different from the planar or concave because the combined effect of heat conduction from solidified airfoil, the radiant heat transfer of the melt in the platform ends to furnace chamber and the geometry-related hot spot in the middle of the platform acting as a heat reservoir to prevent the temperature decreasing.
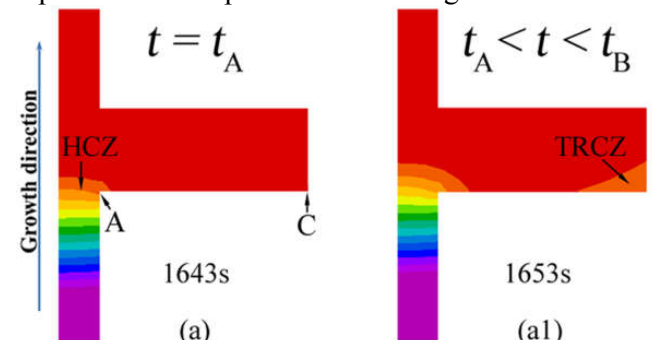

(a)

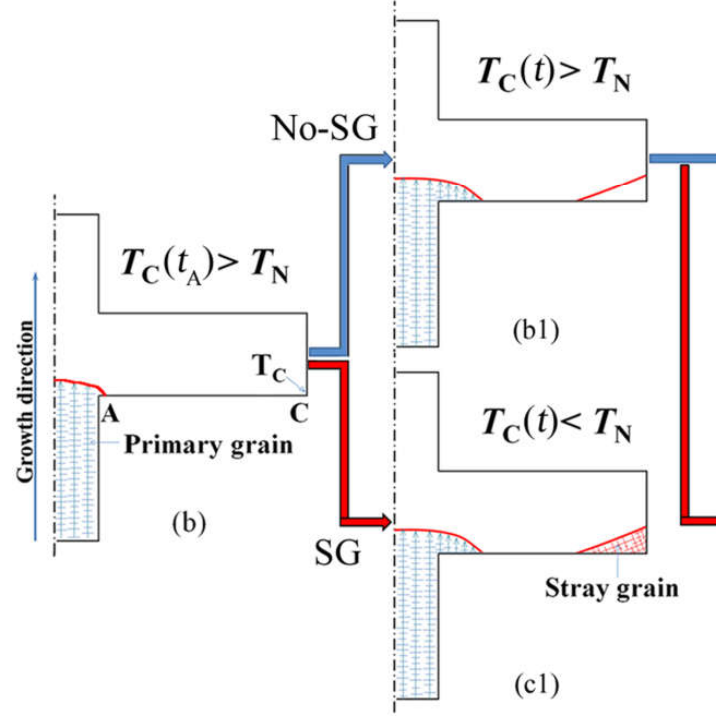

The detailed thermal evolution process is shown in Figure 6 (a)(a3). The platform, when initially located inside of the furnace, is superheated as the radiation heating is intense enough to overcome heat loss. As the platform is gradually withdrawn out of the furnace, the liquidus reaches the intersection of the airfoil and the platform, and then spreads into platform resulting in the HCZ formation (Figure 6 (a)). Afterwards, the radiation cooling begins to take effect in platform ends first as the view factors between these sites and the cool surrounding increase more sharply in comparison to the middle section, resulting in these sites cooling earlier to form a TRCZ. The remainder of the platform with inefficient cooling rate then constitutes HSZ (Figure 6 (a1)). As directional solidification continues, the liquidus gradually connects (Figure 6 (a2)) and advances upwards to leave the platform (Figure 6 (a3)). The effect of such a distributed thermal profile on the dendritic growth pattern in platforms suffering from No-SG and SG are schematically shown in Figure 6 (b)-(b3) and Figure 6 (b)-(c3), respectively. At $t=t_{\mathrm{A}}$, the solidified dendrites just reach the re-entrant of platform (Point $\mathrm{A}$ ); $T_{\mathrm{C}}\left(t_{\mathrm{A}}\right)$ is higher than the heterogeneous nucleation temperature $T_{\mathrm{N}}$, therefore there is no SG formation (Figure 6 (b)). As solidification continues, dendrites start to branch into the platform and the TRCZ emerges at the end of the platform. At $t_{\mathrm{A}}<t<t_{\mathrm{B}}, t=t_{\mathrm{B}}$ and $t>t_{\mathrm{B}}$, two distinct conditions exist; if $T_{\mathrm{C}}(t)>T_{\mathrm{N}}$, dendritic branches can arrive the platform end resulting in SG formation being prohibited (Figure 6 (b1) (b2) (b3)), otherwise SG nucleates at the end of platform (Figure 6 (c1) (c2) (c3)).

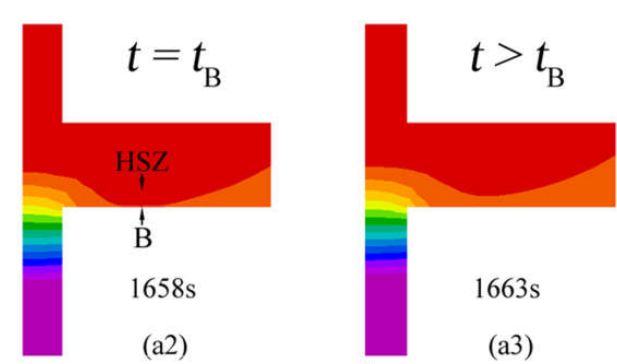

(a2)

(a3)

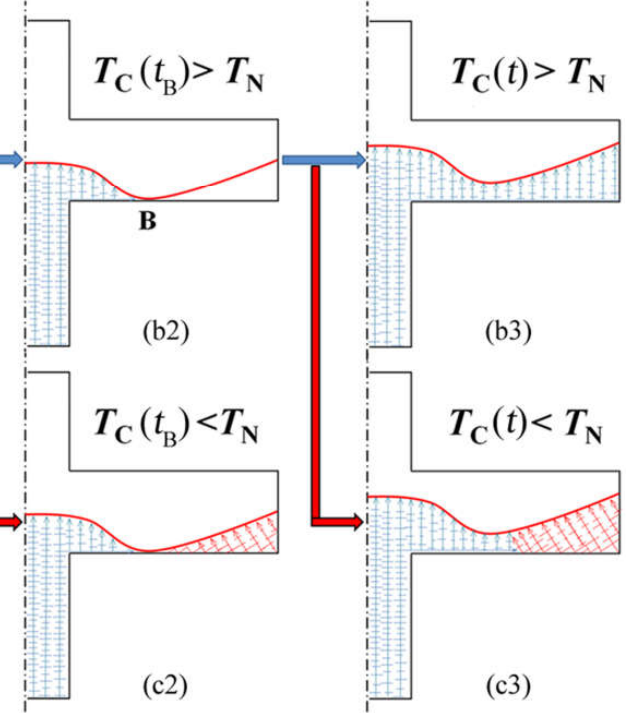

Figure 6. Thermal evolution in the platform ((a), (a1), (a2), (a3)) and its effect on dendritic branching ((b), (b1), (b2), (b3)) and SG formation $((\mathrm{b}),(\mathrm{c} 1),(\mathrm{c} 2),(\mathrm{c} 3)) \cdot t_{\mathrm{A}}$ : the time as the liquidus isothermal just reach the intersection $\mathrm{A} ; t_{\mathrm{B}}$ : the time as the liquidus isothermal connects at point B. $T_{\mathrm{C}}(t)$ : the temperature of point $\mathrm{C}$ at time $t ; T_{\mathrm{N}}$ : the heterogeneous nucleation temperature. 
Criterion of SG Formation. Based on the analyses above, the SG formation can be described as a competitive process between the time of branching from point $\mathrm{A}$ to point $\mathrm{C}$ and the time of cooling of point $\mathrm{C}$ from $T_{\mathrm{C}}\left(t_{\mathrm{A}}\right)$ to heterogeneous nucleation temperature $T_{\mathrm{N}}$ as shown in following equation.

$$
t_{\text {Branch }}^{A \rightarrow C} \geq t_{\text {Cool }}^{T_{C}\left(t_{A}\right) \rightarrow T_{C}^{\text {Nucl }}}
$$

If the time for branching from $\mathrm{A}$ to $\mathrm{C}$ is larger than the time of $\mathrm{C}$ cooling down to heterogeneous nucleation temperature, the SG forms. Otherwise, the SG will be inhibited.
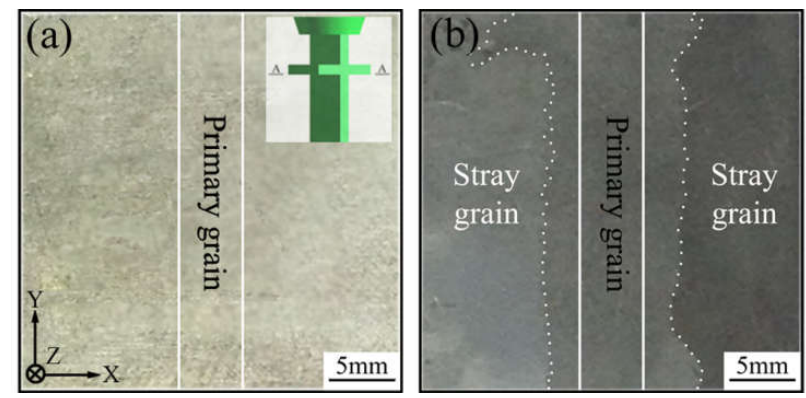

Figure 7. Macroetched platforms fabricated in withdrawal rate case of (a) $3 \mathrm{mmmin}^{-1}$ and (b) $9 \mathrm{~mm} \mathrm{~min}^{-1}$ with other parameters being set as the baseline.

\section{Influencing Factors of SG Formation}

Effect of Withdrawal Rate. The effect of withdrawal rate on SG formation is shown in Figure 7. In $3 \mathrm{~mm}^{-m^{-1}}$ case, the entire macroetched platform features the same light contrast, indicating that SX fills up the platform and no SG forms (Figure 7 (a)).With increasing the withdrawal rate to $9 \mathrm{~mm} \mathrm{~min}^{-1}$, SGs form and occupy almost two-thirds of the entire platform area (Figure 7 (b)), indicating that the high withdrawal rate triggers the SG formation.

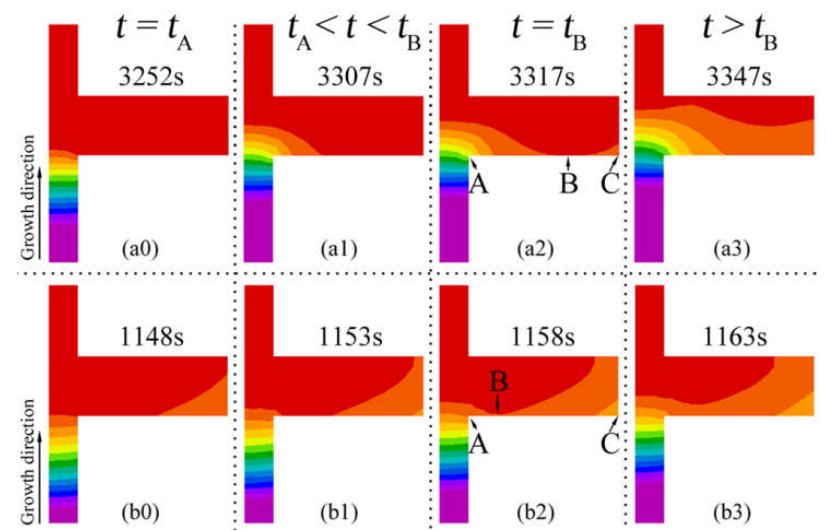

Figure 8 . Thermal evolution in the platform of the different withdrawal rate case: (a0)-(a3) $3 \mathrm{~mm}^{\mathrm{min}} \mathrm{mi}^{-1}$; (b0)-(b3) $9 \mathrm{~mm}^{\mathrm{min}} \mathrm{m}^{-1}$.

By analyzing the thermal evolution in different cases (Figure 8), the explanation could be made as follows: TRCZ does not exist initially in the low withdrawal rate case (Figure 8 (a0), (a1)) but forms when the liquidus connects at point B (Figure 8 (a2)), which then advances upwards to let the dendrites branch into TRCZ (Figure8 (a3)). The small undercooling in TRCZ provides enough time for dendritic branches reaching the platform end, making SG formation thermodynamically impossible. While in the higher withdrawal rate case, the TRCZ has already formed before the dendrites reached the intersection A (Figure 8 (b0)) and then undergoes an extension towards the airfoil section leading the platform end $\mathrm{C}$ even cooler (Figure 8 (b1) (b2)). The heterogeneous nucleation occurs before the high temperature valley leaves the platform base (Figure 8 (b3)) to let the branches get through.
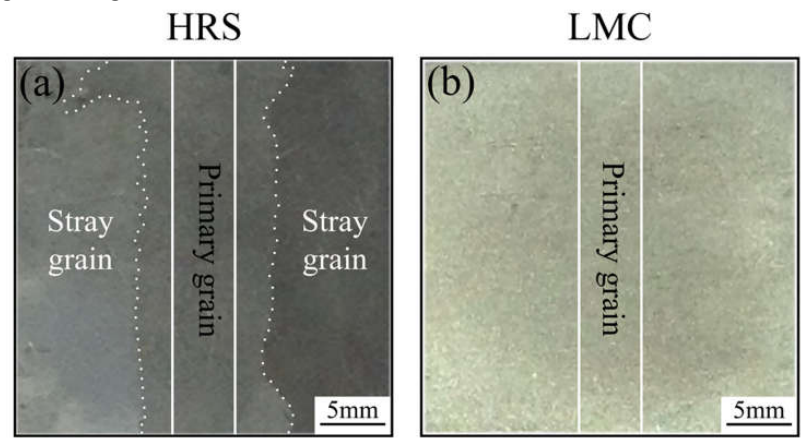

Figure 9. Macroetched platforms fabricated by different directional solidification methods at $V=9 \mathrm{mmmin}^{-1}$ with other parameters being set as the baseline.

Effect of Directional Solidification Method. The effect of directional solidification method on SG formation is shown in Figure 9. LMC technique can effectively avoid SG formation (Figure 9 (b)) in comparison to HRS technique (Figure 9 (a)). The thermal evolution in the platform using the LMC technique is comparatively shown in Figure 10 with that of HRS at the same withdrawal rate of $9 \mathrm{~mm} \mathrm{~min}^{-1}$. Enhanced heat conduction through the airfoil section by heat convection between the ceramic mold and the liquid metal can effectively ease the effect of HSZ and renders the dendrites to branch into the platform earlier, before the platform ends are thermally undercooled (Figure 10 (b0), (b1)). Thereafter, the formed TRCZ as shown in Figure 10 (b2) with small undercooling cannot cool fast enough to let the SG nucleate before the dendrites reach C from B (Figure 10 (b3)), making the SG formation impossible.

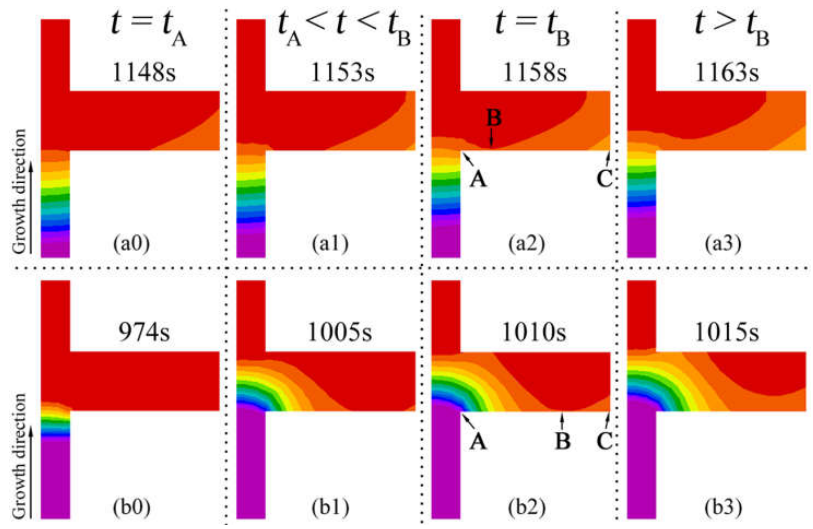

Figure 10. Thermal evolution in the platform of the different solidification method: (a0)-(a3) HRS; (b0)-(b3) LMC.

Effect of Platform Dimension. The effect of platform dimension on SG formation is shown in Figure 11. The tendency of SG formation increases with the increase of the platform dimension. 
The results could be explained from the facts that the larger platform necessitates the dendrites to branch a longer distance to fill up the entire platform region and the larger platform end features a larger radiation angle to the cooling ambient such that it cools more quickly. This is confirmed by simulated thermal profiles of different types of platforms as illustrated in Figure 12. The features of the thermal contours are the same in three cases with the only difference that the large platform is largely undercooled in the platform end relative to the small one (comparing Figure 12 (c1), (c2), (c3) with (a1), (a2), (a3)). The larger undercooling in the platform end leads to a reduced cooling time from $T_{\mathrm{C}}$ to $T_{\mathrm{N}}$. Besides, the large platform necessitates a long time for dendrites to reach the platform end. These two factors together render the large platform possessing a greater tendency to suffer SG.

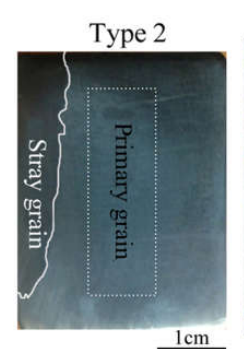

(a)

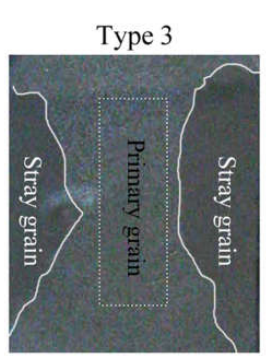

(b)

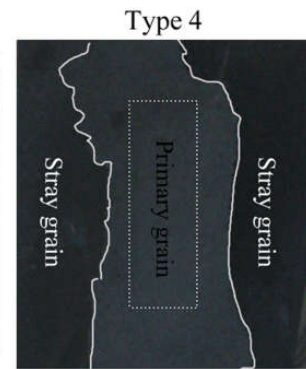

(c)
Figure 11. Macroetched platforms with different dimensions fabricated at $V=6 \mathrm{~mm}^{-1} \mathrm{~min}^{-1}$ with other parameters being set as the baseline.

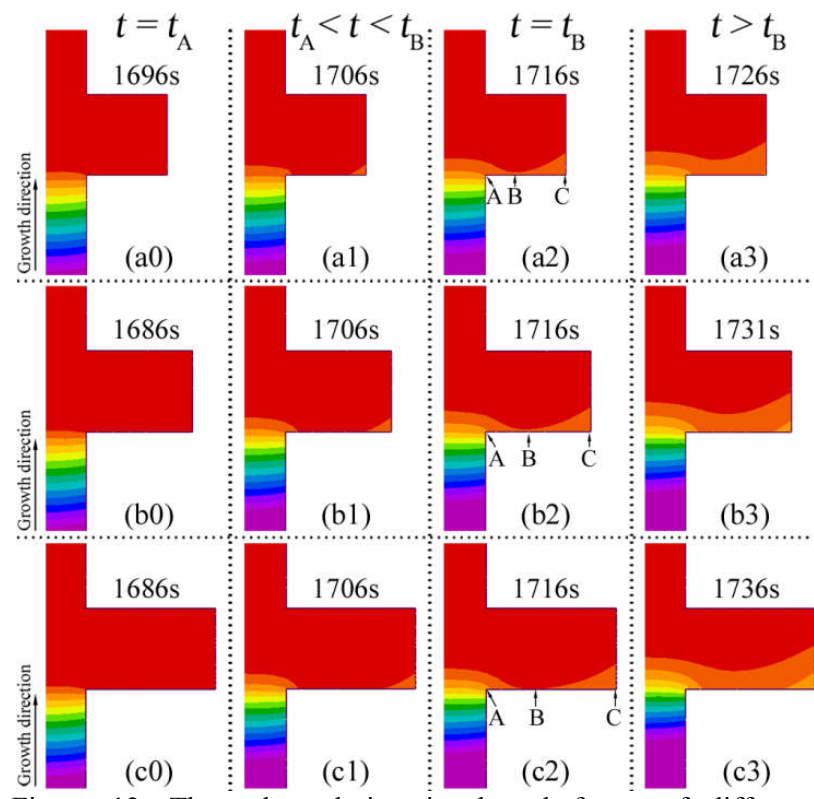

Figure 12. Thermal evolution in the platforms of different dimensions: (a0)-(a3) Type 2; (b0)-(b3) Type 2; (c0)-(c3) Type 4.

Effect of Alloying Elements. Figure 13 shows the experimental results of the dependences of SG on different alloying systems. Refractory element Re has little effect on SG formation as only LABs form in Re alloys due to the dendritic deformation during branching process instead of SG nucleation in the present study (Figure 13 (a1) (a2)). The grain boundary strengtheners C and B affect the SG emergence such that increasing the content of these two elements lowers the propensity of SG formation (Figure 13 (b1), (b2), (c1), (c2), (c3)). As mentioned above, SG formation is a competitive process between the time of dendritic branching and the time of thermal undercooling nucleation. The different impacts of the individual alloying elements on SG formation can be interpreted according to their different impacts on branching time and nucleation temperature.
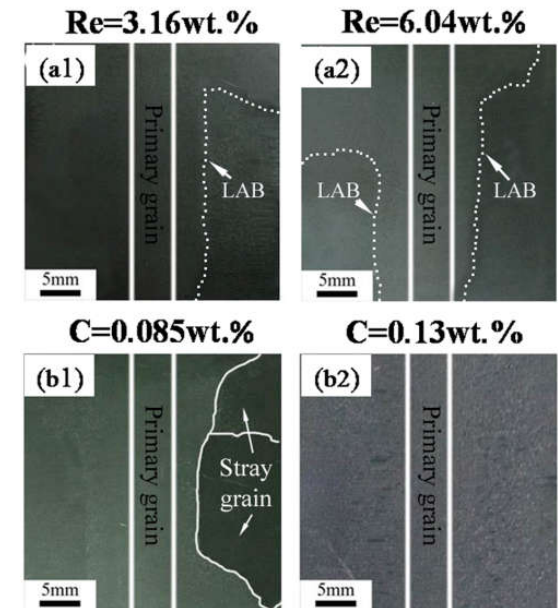

\section{$\mathrm{B}=0.000 \mathrm{wt} . \%$}
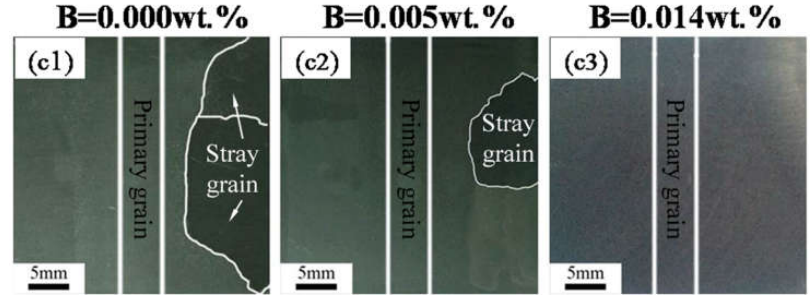

Figure 13. Macrostructures of the macroetched platforms fabricated using different alloys at $V=6 \mathrm{mmmin}^{-1}$ with other parameters being set as the baseline.
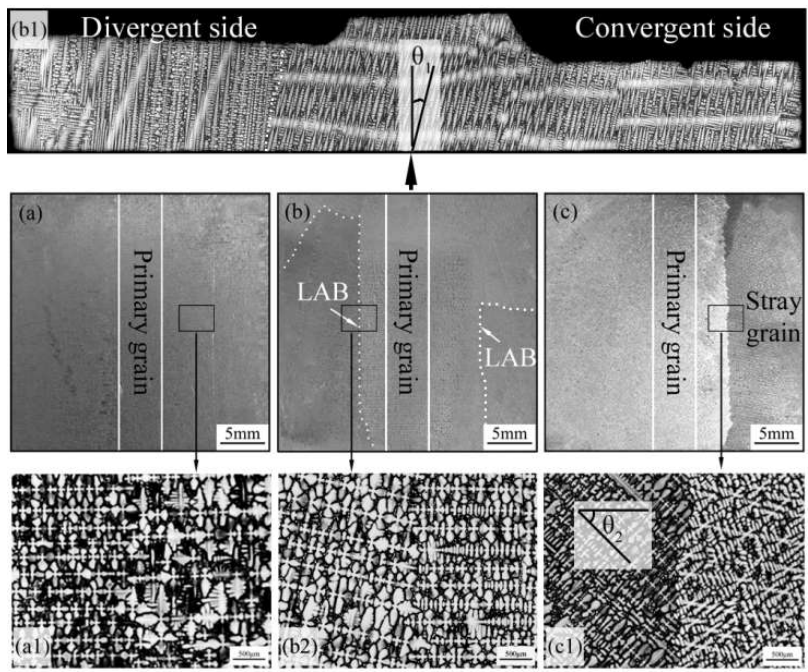

Figure 14. Macro- and microstructures of the platforms fabricated using specially oriented seeds of (a) $\left(\theta_{1}, \theta_{2}\right)=\left(0^{\circ}, 0^{\circ}\right)$, (b) $\left(\theta_{1}, \theta_{2}\right)$ $=\left(15^{\circ}, 0^{\circ}\right)$ and $(\mathrm{c})\left(\theta_{1}, \theta_{2}\right)=\left(0^{\circ}, 45^{\circ}\right)$ at $V=6 \mathrm{~mm}^{\prime} \mathrm{min}^{-1}$ with other parameters being set as the baseline. 
Effect of Grain Orientation. The effect of primary and secondary orientation on SG formation is shown in Figure 14. In comparison to No-SG case of $\left(\theta_{1}, \theta_{2}\right)=\left(0^{\circ}, 0^{\circ}\right)$ in Figure 14 (a) and (a1), the $15^{\circ}$ inclination of primary dendrites to the platform does not trigger the SG formation. Only LABs form both in the divergent side and the convergent side (Figure 14 (b), (b1), (b2)) because the wave-shaped thermal contour forms an included angle with oriented dendrites leading to the asymmetrical branching and shrinkage in mush zone, which causes dendritic deformation resulting in the LAB formation. SG forms in the case of $\left(\theta_{1}, \theta_{2}\right)=$ $\left(0^{\circ}, 45^{\circ}\right)$ where secondary orientation is rotated $45^{\circ}$ around $\mathrm{Z}$-axis from the $+\mathrm{X}$ direction ((Figure 14 (c), (c1)). This could be simply explained as the rotation elongating the branching distance from A to $\mathrm{C}$ leading to more time for $\mathrm{C}$ to cool down to the nucleation temperature to form SG (Figure 15).

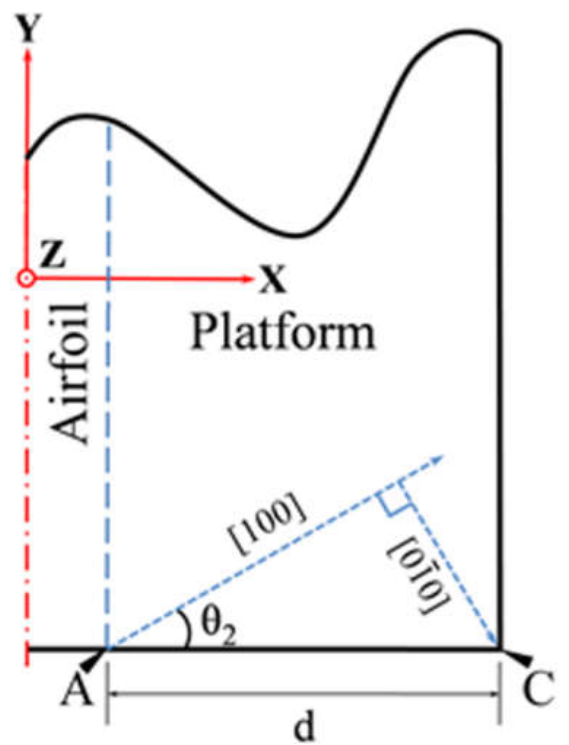

Figure 15. Schematic of dendritic growth with the secondary orientation deviation from coordination system.

\section{$\underline{\text { SG Controlling Techniques }}$}

The technical details of SG controlling methods, the experimental results and the simulated thermal evolution processes are shown in Figure 16 with the $9 \mathrm{mmmin}^{-1}$ withdrawal rate case as the baseline for comparison. In contrast to the baseline situation with SG forming in the platform (Figure 16 (a1)), the three SG controlling methods can all effectively inhibit the SG occurrence (Figure 16 (b1), (c1) (d1)) with different mechanisms.

Withdrawal rate deceleration (WRD) technique by decreasing the withdrawal rate after a period of withdrawing at constant speed postpones the formation of TRCZ (Figure 16 (b2)) and earns enough time for dendrites branching to the platform ends and therefore prevents the SG formation (Figure 16 (b3), (b4), (b5)).

As to grain continuator (GC) technique, two additional cylinder bars are added to connect airfoil section and platform ends (Figure 16 (c). The assistant bars act as bypass channels to let the SX get through to fill up the platform ends before the nucleation happens, resulting in SG being inhibited (Figure $16(\mathrm{c} 1)$ ). The thermal evolution process confirms that the dendrites first arrive at the platform ends (Figure $16(\mathrm{c} 1)$ ) and then spread towards airfoil to finally full up the entire platform (Figure 16 (c3), (c4), (c5)).

The graphite heat conductor (GHC) technique (Figure16 (d)) can effectively eliminate the SG formation, as shown in Figure16 (d1) that GHC side has no SG formation, while the other side without GHC adding SG occurs. This is mainly because the GHC starts to export heat before the solidified dendrites reach A (Figure 16 (d2)) leading to the HSZ being effectively diminished (Figure 16 (d2), (d3)) and leveling the comparative wave-shaped isothermal (Figure16 (d4)) to make the branching much easier to the platform ends to prevent SG formation (Figure $16(\mathrm{~d} 5)$ ).

Additionally, the SG could be controlled by using the LMC directional solidification technique, by adjusting the minor elements of Carbon or (and) Boron or by precisely controlling the crystal orientation as discussed in the influencing factors section.

\section{Conclusion}

1. The liquidus isothermal in the platform region during directional solidification process is wave-shaped rather than planar or concave. This wave-shaped isothermal affects the dendritic branching process and the resulting SG formation process. The criterion for SG formation is expressed as a competition between the time of dendrites branching from the airfoil to the platform end and the time of cooling of platform end to the heterogeneous nucleation temperature.

2. The following factors have different impacts on SG formation:

(1) Increasing the withdrawal rate results in easier SG formation.

(2) The LMC technique can effectively inhibit the SG nucleation.

(3) The larger platform dimension increases tendency to form SG.

(4) Rhenium does not affect the SG formation in the present study, but the increased content of Carbon and Boron can eliminate SG formation.

(5) With the increase of the deviation angle $\theta_{2}$, the proneness of SG formation increases.

3. The SG controlling techniques can effectively inhibit SG formation by changing the local thermal profile within platform region to diminish the extent of undercooling in platform ends or by promoting the dendritic branching from the airfoil section to the platform ends.

\section{Acknowledgments}

The authors are grateful to the financial support from the National 973 Program (2011CB610406), National 863 Project (2012AA03A511), the National Natural Science Foundation of China (51171161, 51331005, 51601161), Natural Science Foundation of Shaanxi Province (2014JM6227) and Supported by the fund of the State Key Laboratory of Solidification Processing in NWPU (SKLSP201407). 


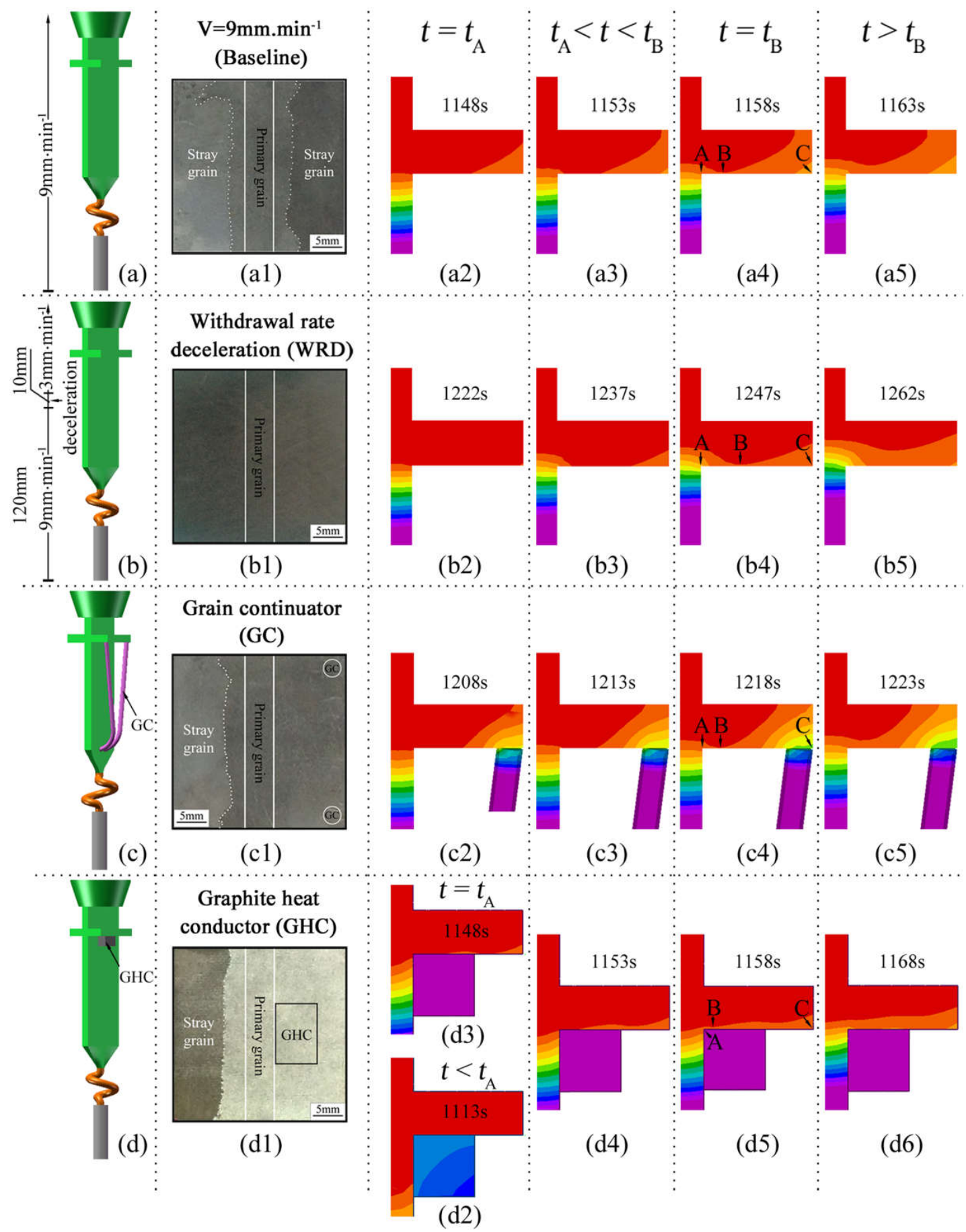

Figure 16. Different SG controlling techniques and their effects on SG formation and thermal evolution at condition of $V=9 \mathrm{~mm}^{\mathrm{min}}{ }^{-1}$ and other parameters being set as the baseline.

\section{References}

1. Roger C. Reed, The Superalloys: Fundamental and Applications (Cambridge, UK: Cambridge University Press, 2006), 1-5.

2. B.B. Seth, "Superalloys-The Utility Gas Turbine Perspective," Superalloys 2000, ed. T.M. Pollock et al. (Warrendale, PA: TMS, 2000), 3-16.

3. A.D. Bussac and C.A. Gandin, "Prediction of A Process Window for the Investment Casting of Dendritic Single Crystals", Mater Sci Eng A, 237 (1997), 35-42. 
4 M. Meyer ter Vehn et al., "Undercooling Related Casting Defects in Single Crystal Ni-Based Superalloy," Superalloys 1996, ed. R.D. Kissinger et al. (Warrendale, PA: TMS, 1996), 471-479

5 X.L. Yang et al., "Microscale Simulation of Stray Grain Formation in Investment Cast Turbine Blades," Mater Sci Eng A, 386 (2004), 129-139.

6 D. Ma, Q. Wu, and A.B. Polaczek, "Undercoolability of Superalloys and Solidification Defects in Single Crystal Components," Adv Mater Res, 278 (2011), 114-19

7. X.L. Zhang et al, "Study on the Tendency of Stray Grain Formation of Ni-Based Single Crystal Superalloys," Acta. Metal. Sinica, 48 (2012), 1229-1236.

8. M.B. Meng et al, "Effect of Platform Dimension on the Dendrite Growth and Stray Grain Formation in A Ni-Base Single Crystal Superalloy," Metall and Mater Trans A, 44 (2013), 1955-1965.

9. S.F. Gao et al., "Simulation of stray grain formation at the platform during Ni-base single crystal superalloy DD403 casting," China Foundry, 12 (2015), 118-122.

10. D.X. Ma and A.B. Polaczck, "Application of A Heat Conductor Technique in the Production of Single-Crystal Turbine Blades," Metall and Mater Trans B, 40 (2009), 738748 .

11. M.B. Meng et al., "Method of Stray Grain Inhibition in the Platforms With Different Dimensions During Directional Solidification Of A Ni-Base Superalloy," Metall and Mater Trans A, 45 (2014), 1230-1236.

12. Y.F. Li et al., "The Process Analysis of Seeding-Grain Selection and Its Effect on Stray Grain and Orientation Control," J Alloys Compd, 657 (2016), 341-347

13. A.J. Elliot and T.M. Pollock, "Thermal Analysis of the Bridgman and LMC Directional Solidification Investment Casting Processes," Metall and Mater Trans A, 38 (2007), 871-882

14. J.D. Miller and T.M. Pollock, "The Effect of Processing Conditions on Heat Transfer During Directional Solidification via the Bridgman and Liquid Metal Cooling Processes," Metall and Mater Trans A, 45 (2014), 411-425 